Article

\title{
A Micro-Preconcentrator Combined Olfactory Sensing System with a Micromechanical Cantilever Sensor for Detecting 2,4-Dinitrotoluene Gas Vapor
}

\author{
Myung-Sic Chae ${ }^{1,2, \dagger}$, Jinsik Kim ${ }^{1, \dagger}$, Yong Kyoung Yoo ${ }^{1,3}$, Ji Yoon Kang ${ }^{1}$, Jeong Hoon Lee ${ }^{3, *}$ \\ and Kyo Seon Hwang 1,*
}

1 Center for BioMicrosystems, Korea Institute of Science and Technology, Seoul 136-791, Korea;

E-Mails: bechu88@gmail.com (M.-S.C.); lookup2@hanmail.net (J.K.);

yongkyoung0108@gmail.com (Y.K.Y.); jykang@kist.re.kr (J.Y.K.)

2 School of Electrical Engineering, Korea University, Seoul 136-701, Korea

3 Department of Electrical Engineering, Kwangwoon University, Seoul 139-701, Korea

$\dagger$ These authors contributed equally to this work.

* Authors to whom correspondence should be addressed; E-Mails: jhlee@kw.ac.kr (J.H.L.); kshwang@kist.re.kr (K.S.H.); Tel.: +82-2940-8372 (J.H.L); +82-2958-6727 (K.S.H.).

Academic Editor: Michael Tiemann

Received: 17 June 2015 / Accepted: 23 July 2015 / Published: 24 July 2015

\begin{abstract}
Preventing unexpected explosive attacks and tracing explosion-related molecules require the development of highly sensitive gas-vapor detection systems. For that purpose, a micromechanical cantilever-based olfactory sensing system including a sample preconcentrator was developed to detect 2,4-dinitrotoluene $(2,4-\mathrm{DNT})$, which is a well-known by-product of the explosive molecule trinitrotoluene (TNT) and exists in concentrations on the order of parts per billion in the atmosphere at room temperature. A peptide receptor (His-Pro-Asn-Phe-Ser-Lys-Tyr-Ile-Leu-His-Gln-Arg) that has high binding affinity for 2,4-DNT was immobilized on the surface of the cantilever sensors to detect 2,4-DNT vapor for highly selective detection. A micro-preconcentrator ( $\mu$ PC) was developed using Tenax-TA adsorbent to produce higher concentrations of 2,4-DNT molecules. The preconcentration was achieved via adsorption and thermal desorption phenomena occurring between target molecules and the adsorbent. The $\mu \mathrm{PC}$ directly integrated with a cantilever sensor and enhanced the sensitivity of the cantilever sensor as a pretreatment tool for the target vapor. The response was rapidly saturated within $5 \mathrm{~min}$ and
\end{abstract}


sustained for more than 10 min when the concentrated vapor was introduced. By calculating preconcentration factor values, we verified that the cantilever sensor provides up to an eightfold improvement in sensing performance.

Keywords: DNT; gas sensor; cantilever; micro-preconcentrator

\section{Introduction}

Many research groups have developed highly sensitive and selective sensing or detecting platforms for the explosive materials used to make immediate explosive devices (IEDs), such as trinitrotoluene (TNT), cyclotrimethylenetrinitramine (RDX), and pentaerythritol tetranitrate (PETN). These chemicals have extremely low vapor pressures at concentrations on the order of parts per million (ppm) to parts per trillion (ppt) in the atmosphere at room temperature. Detection of an infinitesimal quantity of these dangerous vapor molecules requires sensors with high sensitivity, high accuracy, and rapid response. Methods for improving the sensitivity and response time, such as the use of a high affinity target molecule receptor and additional pretreatment techniques, have been employed in previous sensor systems.

Improvements in micro/nano electromechanical systems (MEMS/NEMS) technology have led to the development of various miniaturized mechanical sensors for chemical and biological applications requiring high sensitivity. The cantilever sensor is the most widely used promising micromechanical sensor owing to its excellent sensing capabilities $[1,2]$. The detection limit of the cantilever sensor is approximately $500 \mathrm{ppt}$, which is sufficient for detecting gas vapors with very low concentration [3]. Cantilevers that are capable of real-time measurement also have short response time, though the response time depends on the reaction between target molecules and the receptors.

Receptors such as antibodies, deoxyribonucleic acid/ribonucleic acid (DNA/RNA) aptamers, or peptides that have a binding specificity make it possible to selectively immobilize the target molecules on the surface $[4,5]$. These specific binding interactions between receptors and target molecules on the sensor surface change the mechanical properties of the cantilevers, and these changes can be observed by analyzing the resonant frequency response in the dynamic mode [6]. Resonant frequency shifts verify the mass loading of specific interactions on the sensor surface. Generally, the mass loading of target molecules is the dominant variable in relation to changing the resonant frequency. A dynamic cantilever is preferable in terms of quantitative analysis for small molecules owing to its robustness against external disturbance and noise [7,8]. Measuring the resonant frequency change in the dynamic mode of a cantilever sensor is effective also for chemical-vapor monitoring [3,9].

Although cantilever sensors provide sufficiently high sensitivity for detection of extremely low vapor pressures in chemical gas-mixture sensing applications, such as when explosive or volatile organic compounds (VOCs) in the atmosphere must be detected, it is possible to further enhance the sensing performance. This enhancement can be achieved using a preprocessing procedure for the sample, such as a preconcentration step, in order to improve sensitivity and the detection limit. Preconcentration is commonly used as a preprocessing tool for gas-mixture analysis; it improves quantitative analytic performance by trapping analytes and releasing them into a detector as an enriched plug. Using MEMS 
fabrication technology, it is possible to develop a compact micro-preconcentrator ( $\mu \mathrm{PC})$ that achieves high concentration efficiency $[10,11]$.

Here, we introduce a cantilever-based chemical-vapor detection system combined with a $\mu \mathrm{PC}$ for detecting ultra-low concentrations of 2,4-dinitrotoluene (2,4-DNT) vapor, which is a by-product and trace marker of the explosive molecule TNT. The 2,4-DNT molecule exists in concentrations of 160 parts per billion ( $\mathrm{ppb}$ ) at room temperature at atmospheric pressure. The fabricated microcantilever sensor is operated by an embedded piezoelectric thin film. The specific sequenced 12-mer peptides were immobilized as receptors for 2,4-DNT vapor on the cantilever surfaces to provide the selectivity of the cantilever sensor. The $\mu \mathrm{PC}$ was also fabricated using the MEMS techniques, and it was coated with an adsorbent polymer to provide structures for trapping volatile explosive-related compounds. The $\mu \mathrm{PC}$ was incorporated ahead of the sensing reaction chamber, where it can accumulate and release target vapor. The volume of the sample matrix and environmental conditions for preconcentration were established by considering compatibility with our cantilever sensor. Comparing the resonant frequency change of cantilever sensors with and without a sample preconcentrator confirmed the expected performance enhancement.

\section{Experimental Details}

A diagram representing the 2,4-DNT gas-vapor detection protocol with the proposed $\mu \mathrm{PC}$-integrated cantilever sensor system is provided in Figure 1. The surface of the cantilever was functionalized by 12-mer peptide receptors for high binding specificity resulting in high selectivity. The binding interaction of 2,4-DNT vapor leads to changes in the resonant frequency of the dynamic actuated cantilever sensor, and the change in resonant frequency is measured. The integrated $\mu \mathrm{PC}$ concentrated the target gas vapor, 2,4-DNT, using Tenax-TA. The concentrated 2,4-DNT gas was desorbed with $270{ }^{\circ} \mathrm{C}$ thermal heating and flowed into the reaction chamber containing the cantilever sensor. The changes in resonant frequency were measured using a real-time electrical detection system.

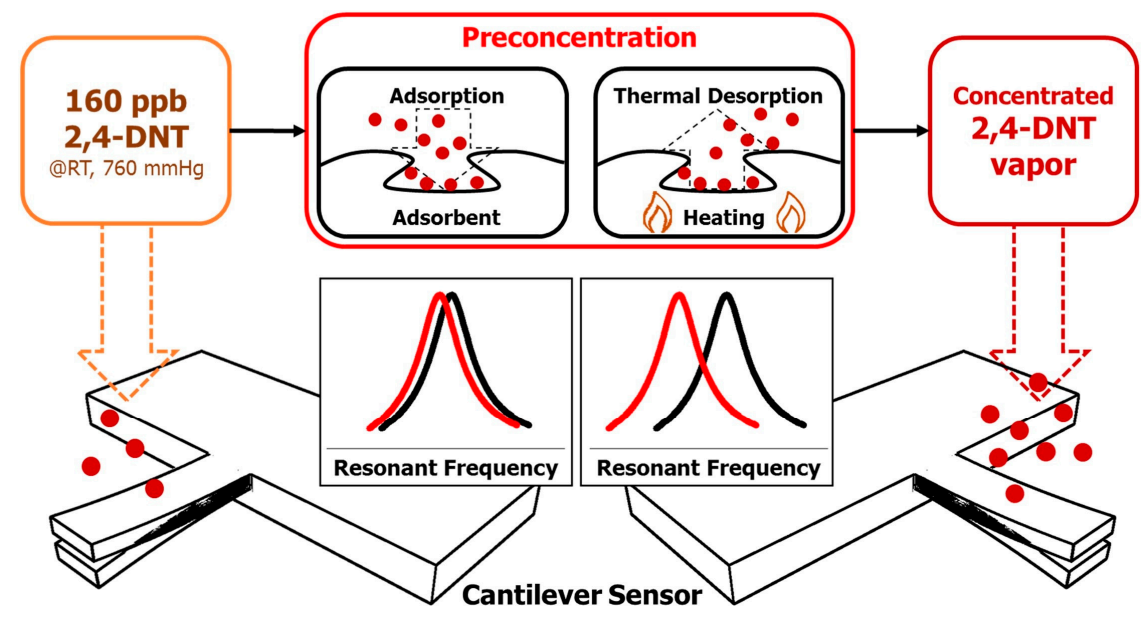

Figure 1. Functional model of the cantilever-based artificial olfactory system with sample preconcentration for 2,4-DNT vapor detection.

The detection system includes a multiarrayed cantilever sensor, a gas-generating system with a $\mu \mathrm{PC}$, and a signal-processing system, as shown in Figure $2 \mathrm{a}$. The gas-generating system consists primarily of 
a pair of mass-flow controllers (MFCs), solenoid valves, and an on/off toggle switch. Continuously injecting nitrogen gas $\left(\mathrm{N}_{2}\right)$ at a 50 standard cubic centimeter per minute $(\mathrm{sccm})$ flow rate provided stabilization of the resonant frequency and 2,4-DNT molecule delivery. The cantilever sensor was located in a reaction chamber connected between the gas-generating and signal-processing systems. The reaction chamber has an inlet and outlet through a sensor chip loading for a gas sample stream that was prepared from polyetheretherketone (PEEK) material [9]. The $\mu \mathrm{PC}$ was located in front of the reaction chamber containing the cantilever sensors, and it was placed on a heater to maintain the thermal desorption temperature. Then, we connected a signal-processing system for measuring the sensor's resonant frequency. The signal-processing system enabled real-time monitoring of changes in the resonant frequency of the sensor due to interactions between the target vapor and the peptide receptors. For measuring electrical signals from the cantilever array, we employed a PC-controlled electrical measurement system (MiCan 2.0, Cantis Corporation, Ansan, Korea) equipped with an analog-to-digital converter (ADC), a charge amplifier, a digital signal processor (DSP), and a digital-to-analog converter (DAC).

We fabricated a multiarrayed micromechanical cantilever sensor with 2.18 - $\mu$ m-thick $\mathrm{SiN}_{\mathrm{x}} / \mathrm{Ta} / \mathrm{Pt} / \mathrm{PZT} / \mathrm{Pt} / \mathrm{SiO}_{2}$ multilayers (see Figure $2 \mathrm{~b}$ ). In order to actuate the cantilever, 1.5- $\mu \mathrm{m}$-thick piezoelectric layer (PZT) was deposited via the sol-gel method; it was intended to function as a self-actuating layer without need for external oscillating equipment. We thus fabricated a dynamic mode cantilever that offers higher Q-factor and sensitivity than a static mode cantilever. According to Equation (1), the resonant frequency $\left(F_{r}\right)$ is determined not only by the inducing mass and surface stress, caused by molecular interaction on the cantilever surface, but also by the thermal events, which can affect the mechanical characteristics owing to the different thermal expansion coefficients of each layer [12-14]:

$$
F r=\frac{1}{2 \pi} \sqrt{\frac{k}{m^{*}}}
$$

where $k$ is the spring constant and $m^{*}$ is the effective mass of the cantilever. In our experimental methods, thermal issues could occur in the 2,4-DNT preconcentration process. A dynamic mode cantilever sensor with embedded piezoelectric actuating layer was designed to avoid this subsidiary effect in order to achieve accurate sensing of low-concentration gas vapors.

The fabrication process for the multi-arrayed cantilever sensors followed that used in previous studies $[9,15]$. The fabricated sensors have four reaction units (indicated by the yellow-dashed-line rectangular area in Figure 2b), and each unit contains three individual cantilevers that operate as an array and a reference cantilever that ensures accurate resonant frequency measurement by reducing parasitic capacitance. An example of the three operable cantilevers in the reaction unit is shown in Figure 2c.

The fabricated piezoelectric cantilever sensors have resonant frequencies of $24.5 \pm 3 \mathrm{kHz}$ and dimensions of $100 \times 300 \mu \mathrm{m}$, and these values are uniform for all the cantilevers in a reaction unit array. To avoid external disturbance during interactions between the target vapor and the peptide receptor on the cantilever sensor surface, the four reaction units can be functionalized for different surface conditions (2,4-DNT specific peptide, 2,4-DNT nonspecific peptide immobilized and peptide-receptor-free surface).

A Si-based $\mu$ PC was fabricated and sealed with a Pyrex glass cover consisting of a gas inlet and outlet and a concentration chamber with dimensions of $10 \times 20 \times 0.3 \mathrm{~mm}$ (width $\times$ length $\times$ thickness) (see Figure 2d). The concentration chamber has a micropattern that increases the surface area for gas-vapor 
adsorption. Sandblasting was used to form inlet and outlet holes for gas collection on the Pyrex glass wafer, and the microstructural Si wafer was bonded with the Pyrex glass wafer via anodic bonding. For the 2,4-DNT concentration used as a sample pretreatment, polymer adsorption and thermal desorption phenomena occurring between the adsorbent and specific molecules were utilized according to a gas-collection method [16]. Tenax-TA is a 2,6-diphenylene oxide-based porous polymer resin. Its capabilities have been validated for sample treatment in gas analysis instruments, such as gas chromatograph-mass spectrometers (GC-MS). Coating the concentration chamber with Tenax-TA (80-100 mesh, Sigma-Aldrich, St. Louis, MO, USA,) makes it possible to selectively preconcentrate the volatile organic analytes of interest $[17,18]$. Tenax-TA adsorbent was dissolved in dichloromethane with a concentration of $10 \mathrm{mg} / \mathrm{mL}$. The microstructures were immersed in an adsorbent solution and washed with methyl alcohol. After evaporation of the solvent, Tenax-TA was deposited as shown in Figure 2e.
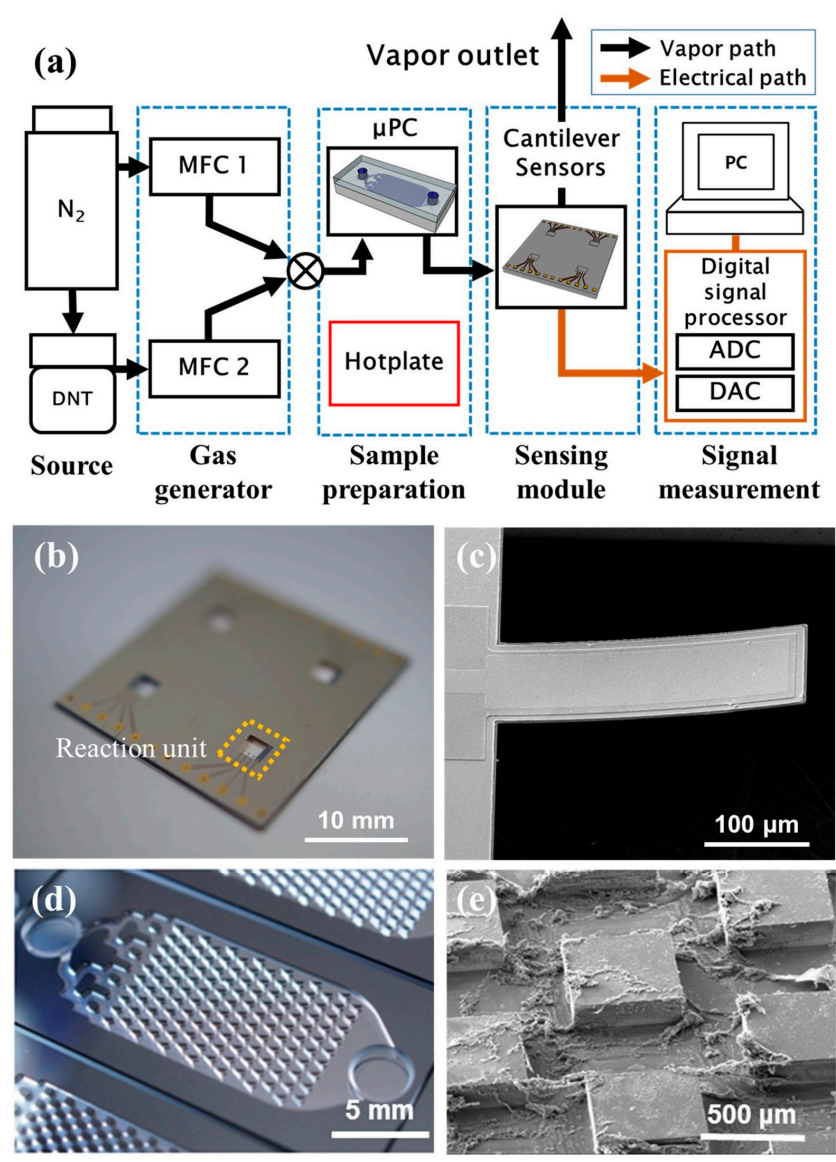

Figure 2. Olfactory sensing system integration with micromechanical cantilever sensors and $\mu \mathrm{PC}$ for detection of 2,4-DNT vapor (a) Schematic of the experimental setup for gas vapor detection with sample preconcentration. (b) Fabricated multiarrayed cantilever sensor with four reaction units. Each unit includes three operable cantilevers. (c) Multilayered single cantilever with dimensions of $100 \times 300 \mu \mathrm{m}$. (d) Fabricated Si-based $\mu$ PCs with microstructures. (e) SEM image of coated adsorbent (Tenax-TA) on the microstructure of $\mu \mathrm{PC}$.

The peptide receptors were immobilized on the bottom surface of the cantilevers. The specific binding peptide (SBP) for the target vapor has a sequence (12-mer, His-Pro-Asn-Phe-Ser-Lys-Tyr-Ile-Leu-His- 
Gln-Arg) determined by phage-display screening to bind 2,4-DNT with high affinity [3]. The non-specific binding peptide (NSP) has randomly allocated sequences (12-mer, Thr-Ser-Met-Leu-LeuMet-Ser-Pro-Lys-His-Gln-Ala) and was immobilized as a control on another cantilever array. At first, the Au layer $(50 \mathrm{~nm})$ was deposited with a Cr adhesive layer $(10 \mathrm{~nm})$ on the rear surface of the cantilever using an e-beam evaporator. The Au surface was cleaned with piranha solution (a 4:1 ratio of $\mathrm{H}_{2} \mathrm{SO}_{4}$ to $\mathrm{H}_{2} \mathrm{O}_{2}$ ) and rinsed with deionized (DI) water to remove potential contaminants. Self-assembled monolayers (SAMs) were formed on the Au surface by immersing a mixed solution with a 9:1 ratio of tri-(ethylene glycol)-alkanethiol (HS-EG3-OH) to carboxyl-terminated penta-(ethylene glycol)-alkanethiol (HS-EG6-COOH) in ethanol for $12 \mathrm{~h}$. The modified Au surface was treated with a mixture of 1-ethyl-3-(3-dimethylaminopropyl) carbodiimide (EDC) and 2,5-pyrroledione (maleimide) dissolved in a phosphate buffered solution (PBS, $\mathrm{pH}=7.4$ ) with $100 \mathrm{mmol} / \mathrm{L}$ and $50 \mathrm{mmol} / \mathrm{L}$, respectively, for $2 \mathrm{~h}$. A peptide-receptor solution with a concentration of $10 \mu \mathrm{g} / \mathrm{L}$ in a PBS was incubated on the surface of the maleimide-functionalized SAMs for $2 \mathrm{~h}$. The cysteine terminal from the thiol group of peptides was conjugated with maleimide to form a thioether linkage. The surface was cleaned with PBS, DI water, and ethyl alcohol for each process.

\section{Results and Discussion}

The sensitivity and selectivity of the cantilever sensors were verified without preconcentration by injection of $160 \mathrm{ppb}$ of 2,4-DNT vapor mixed with $\mathrm{N}_{2}$ gas for $10 \mathrm{~min}$ after stabilization with $50 \mathrm{sccm}$ of $\mathrm{N}_{2}$ gas for $50 \mathrm{~min}$. After a reaction time of $10 \mathrm{~min}$, the reaction chamber was purged with pure $\mathrm{N}_{2}$ at $50 \mathrm{sccm}$. The total volumetric stream of 2,4-DNT vapor was $500 \mathrm{~mL}$. The resonant frequency was immediately changed after target-vapor injection, as shown in Figure 3. The response of the SBP (blue dashed line) and NSP (green dotted line) immobilized cantilever sensors was $-6 \mathrm{~Hz}$ and $-3 \mathrm{~Hz}$, respectively. This $100 \%$ increase in the resonant frequency shift shows that SBP has a higher binding affinity than NSP. The change from net binding reactions (red solid line) was only $-3 \mathrm{~Hz}$. The values were measured via the differential frequency shift of SBP and NSP, and the difference in resonant frequency was saturated and stabilized within 5 min. After purging with $\mathrm{N}_{2}$ gas, the resonant signal recovered to its normal state. The changes demonstrate that the binding affinity of the peptide receptor leads to resonant frequency changes that are caused by 2,4-DNT gas vapor and that the resonant frequency returns to its normal state when the target gas is removed. Our previous study demonstrated similar frequency shift trends for this differential signal [9].

Prior to evaluating sample preconcentration with a cantilever sensor, it is necessary to define the thermal effects on a multilayered cantilever sensor. Basically, the different volumes of 2,4-DNT vapor were collected via direct injection into the $\mu \mathrm{PC}$ with constant $50 \mathrm{sccm}$ volumetric flow and released via thermal desorption to form a plug stream. The adsorption of the 2,4-DNT is an exothermic chemical reaction that occurs at room temperature with adsorbent. In order to form a highly enriched 2,4-DNT plug, the $\mu \mathrm{PC}$ with adsorbent was rapidly heated by a heater set to $270{ }^{\circ} \mathrm{C}$ to achieve an endothermic desorption process. When the heated 2,4-DNT plug is introduced into the sensor with $50 \mathrm{sccm} \mathrm{N}_{2}$ gas, thermal issues can cause frequency fluctuations in the multilayered cantilever sensors by affecting the difference in the thermal expansion coefficients of each layer. Thus, the heating temperature and time for thermal desorption were optimized to avoid this signal interference. To investigate responses due to 
thermal effects, the cantilever sensor was heated directly from room temperature to $50{ }^{\circ} \mathrm{C}$. The resonant response indicated that our multilayered cantilever has a temperature dependence of $4.6 \mathrm{~Hz} /{ }^{\circ} \mathrm{C}$, as shown in Figure S1 (see Supplementary Information). In regard to eliminating the thermal effects of the endothermic process in preconcentration, we determined that maintaining the sensor at $270{ }^{\circ} \mathrm{C}$ for a minimum of $2.5 \mathrm{~min}$ with a $50 \mathrm{sccm}$ injection of pure $\mathrm{N}_{2}$ gas would sustain stable operation and satisfy the thermal desorption conditions (see Figure S2).

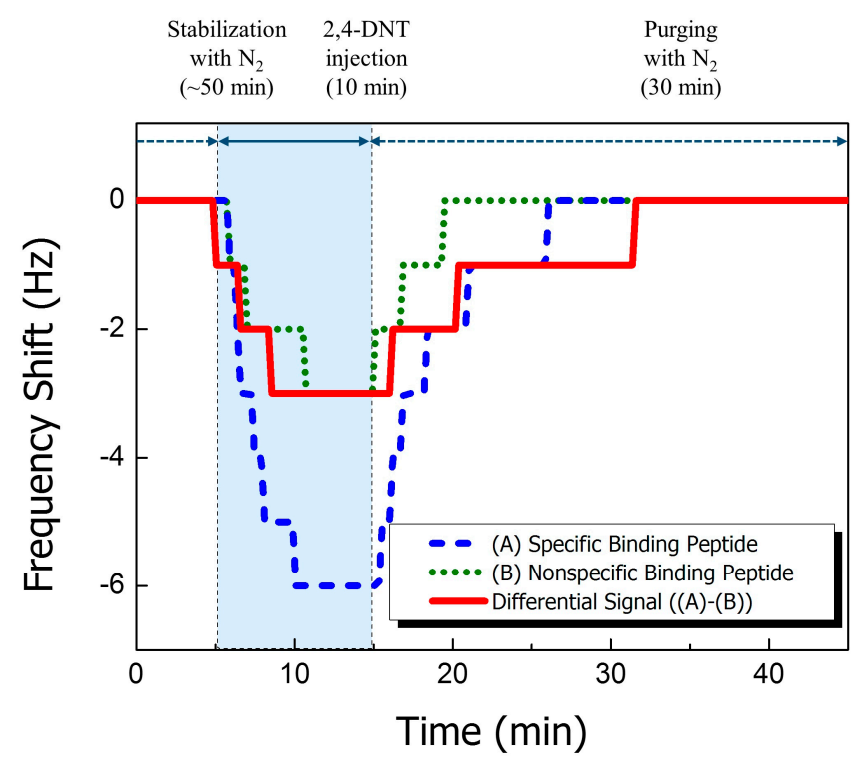

Figure 3. 2,4-DNT differential frequency responses (red solid line) between cantilevers functionalized with specific binding peptide receptors (blue dashed line) and nonspecific binding peptide receptors (green dotted line) in an environment containing $160 \mathrm{ppb}$ 2,4-DNT.

Next, resonant frequency shifts according to the volumes of $160 \mathrm{ppb}$ 2,4-DNT vapor collection (500 mL and $2000 \mathrm{~mL}$ ) were measured to evaluate the performance enhancement of the cantilever sensor with sample preconcentration. As shown in Figure 4a, differential signals between SBP and NSP arrays of $500 \mathrm{~mL}$ and $2000 \mathrm{~mL}$ were obtained as $-4 \mathrm{~Hz}$ and $-8 \mathrm{~Hz}$, respectively. With a preconcentration of $500 \mathrm{~mL}$ 2,4-DNT, the resonant frequency decreased for $10 \mathrm{~min}$ after sample desorption and recovered under continuous $\mathrm{N}_{2}$ flow. A 2,4-DNT volume of $2000 \mathrm{~mL}$ is sufficient to compensate for potential physisorption and allows for fully saturated trapping in a $\mu \mathrm{PC}$ in the limited space of the reaction chamber. The signal for preconcentrated 2,4-DNT vapor with a volume of $2000 \mathrm{~mL}$ decreased more dramatically than in the environment with lower preconcentration. Whereas the volume of 2,4-DNT vapor collected was four times larger, the signal response doubled compared to previous experiments. The signal was rapidly saturated within $3.5 \mathrm{~min}$ via thermal desorption of the concentrated sample, and the saturated condition was sustained for $10 \mathrm{~min}$. Then, the area under the differential signal was four times larger than with the $500 \mathrm{~mL}$ preconcentration condition. This provided more time for recovering the resonant responses. These resonant responses indicate that a higher concentration of 2,4-DNT vapor than is found in the atmosphere was successfully formed and carried into the functionalized cantilever arrays. Based on the response in an environment containing $160 \mathrm{ppb}$ of 2,4-DNT without the $\mu \mathrm{PC}$, the estimated concentration of preconcentrated 2,4-DNT vapor was $213 \mathrm{ppb}$ and $426 \mathrm{ppb}$ for sampling volumes of $500 \mathrm{~mL}$ and $2000 \mathrm{~mL}$, respectively. This shows that more binding interaction occurred in the 
larger quantitative molecules in the preconcentration environment. These concentration values were used to determine the preconcentration factor $(\mathrm{PF})$. The preconcentration performance is generally defined as:

$$
P F=\frac{C_{f}}{C_{0}}
$$

where $C_{0}$ is the concentration of analytes before sample pretreatment and $C_{f}$ is the concentration of preconcentrated analytes. The PF is commonly used as a parameter for evaluating $\mu \mathrm{PC}$ performance as determined by experimental conditions and detectors. The PF value indicates the signal amplification in the detector based on preconcentration with conventional gas chromatography techniques [19,20]. Therefore, we used the ratio of the maximum concentration of trapped 2,4-DNT vapor and the concentration of normally injected target vapor without preconcentration. In a previous study, the concentration was estimated based on the linear relationship between 2,4-DNT concentration and frequency shift [3]. The sample volume for calculating the concentration was determined by the thermally desorbed 2,4-DNT vapor. The PF values for the $500 \mathrm{~mL}$ and $2000 \mathrm{~mL}$ sample volume were calculated as 4 and 12, respectively (see Figure 4b). The trend of the resonant frequency response follows that of the PF values according to volume, as shown in Figure $4 \mathrm{~b}$.

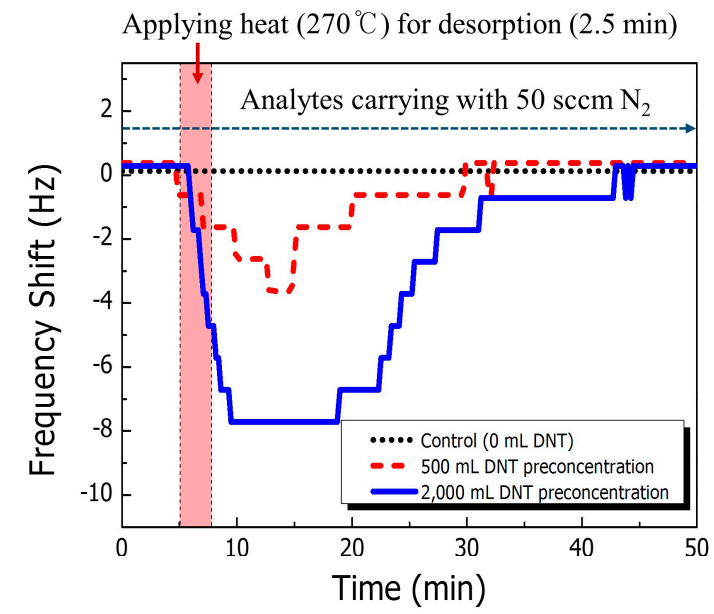

(a)

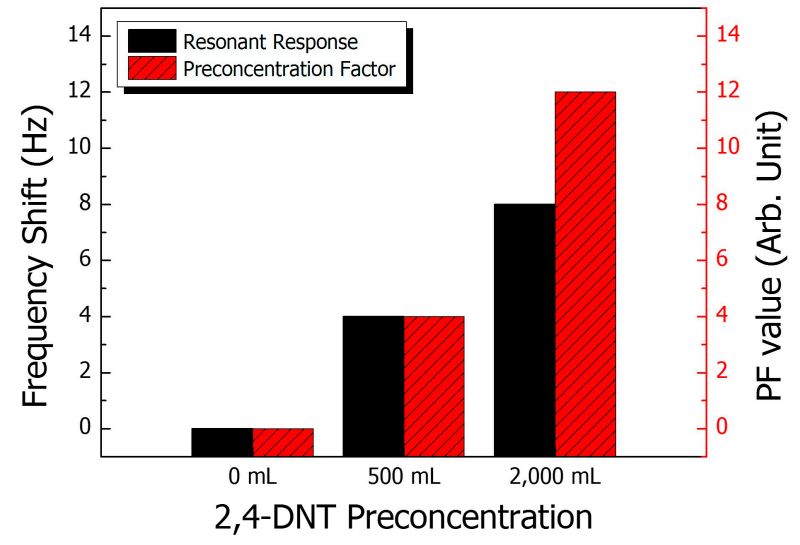

(b)

Figure 4. Performance evaluation for sample preconcentration. (a) Resonant response with 0,500 , and $2000 \mathrm{~mL}$ 2,4-DNT vapor preconcentration for the cantilever sensor with negative control. (b) Frequency shift and preconcentration factor for each preconcentration condition.

Figure 5 shows the effect of the preconcentrator in DNT detection with real-time monitoring. The resonant frequency shift occurred with $160 \mathrm{ppb}$ 2,4-DNT gas for both cases (with and without preconcentration). However, the resonant frequency change with preconcentration is clearly improved relative to that without preconcentration under the same real-time monitoring conditions; the frequency change was approximately three times larger. The calculated PF values suggest that the sensing performance of a cantilever sensor can be enhanced eightfold relative to what was achieved in previous research. Although there are some differences between our theoretical approaches and experimental results, the enhancements are clearly demonstrated, and the system can achieve a sub-ppt detection limit. 


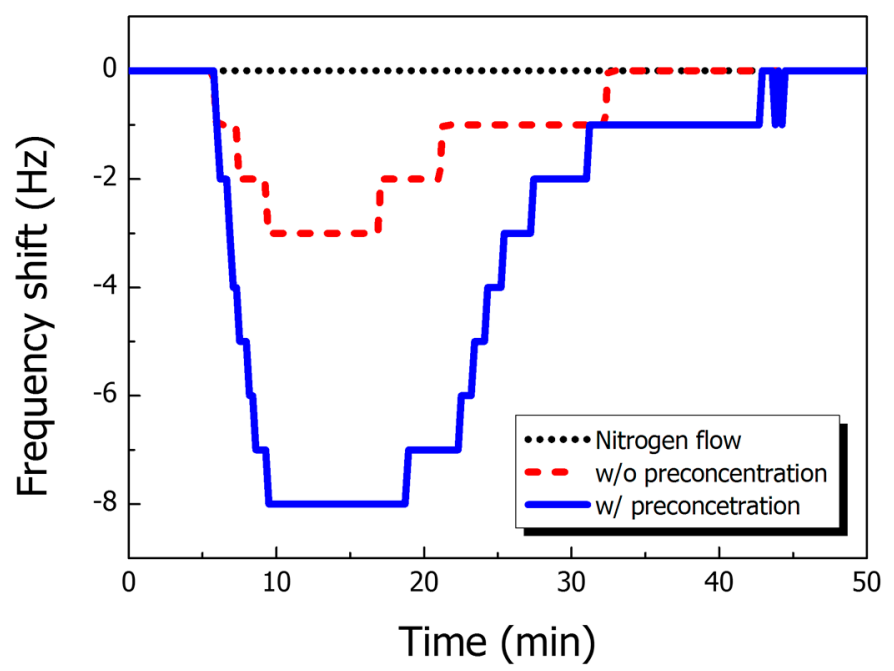

Figure 5. Relative signal response with different sample preprocessing environments: pure $\mathrm{N}_{2}$ flow (black dotted line), direct injection of $160 \mathrm{ppb}$ 2,4-DNT (red dashed line), and 2,4-DNT sample preprocessing with $\mu \mathrm{PC}$ (blue solid line).

\section{Conclusions}

In this paper, we confirmed the enhanced gas-molecule detection sensing performance with 2,4-DNT vapor molecules can be achieved by equipping cantilever sensors with a $\mu$ PC. Combining these two microfabricated devices makes it possible to develop highly sensitive and selective miniaturized gas-analysis systems. Prior to the sample pretreatment evaluations, real-time monitoring of the resonant frequency verified that immobilizing the specific binding peptide on the surface of a micromechanical cantilever sensor provides accurate discrimination between the target molecule and a nonspecific binding peptide. We demonstrated that our $\mu \mathrm{PC}$ is effective, and our cantilever detected 2,4-DNT vapor and exhibited enhanced sensing performance. We used a $\mu \mathrm{PC}$ with an adsorbent coating for sample pretreatment and located it in front of the cantilever sensor to collect and release 2,4-DNT vapor, and this approach tripled the amplitude of the differential signal. The system was able to detect concentrations as low as several hundreds of ppb. This sensitivity is similar to or lower than those reported by other researchers [21,22]. However, we can achieve higher sensitivity by more effectively optimizing the gas flow systems and the condition for releasing 2,4-DNT after preconcentration. For practical applications, the size of the system and detection performance in realistic atmospheric conditions must be considered [21,23]. We anticipate that the techniques described in this paper will make it possible to improve the conventional analytic instruments used in laboratories and will be employed in the field in the near future.

\section{Acknowledgments}

The authors are very grateful for financial support from the KIST institutional program (grant nos. 2E25590 and 2E25690) and the Dual Use Technology Center, sponsored by the Defense Acquisition Program Administration. Jeong Hoon Lee was also supported by a Research Grant from Kwangwoon University in 2014. 


\section{Author Contributions}

Myung-Sic Chae performed the experimental measurements and analysis of data. Jinsik Kim contributed at the theoretical approaches and organized the data analysis. Yong Kyoung Yoo and Ji Yoon Kang conceived the experiments and performed the basic performance of the systems. All the authors participated in design of sensor system, experimental measurements and analysis of results. Myung-Sic Chae and Jinsik Kim wrote this article. All of the authors read and approved the final manuscript. Jeong Hoon Lee and Kyo Seon Hwang supervised the work and finally edited the article.

\section{Conflicts of Interest}

The authors declare no conflict of interest.

\section{References}

1. Hwang, K.S.; Lee, S.M.; Kim, S.K.; Lee, J.H.; Kim, T.S. Micro- and Nanocantilever Devices and Systems for Biomolecule Detection. Annu. Rev. Anal. Chem. 2009, 2, 77-98.

2. Arlett, J.L.; Myers, E.B.; Roukes, M.L. Comparative advantages of mechanical biosensors. Nat. Nanotechnol. 2011, 6, 203-215.

3. Hwang, K.S.; Lee, M.H.; Lee, J.; Yeo, W.S.; Lee, J.N.; Kim, K.M.; Kang, J.Y.; Kim, T.S. Peptide receptor-based selective dinitrotoluene detection using a microcantilever sensor. Biosens. Bioelectron. 2011, 30, 249-254.

4. Backmann, N.; Zahnd, C.; Huber, F.; Bietsch, A.; Pluckthun, A.; Lang, H.P.; Guntherodt, H.J.; Hegner, M.; Gerber, C. A label-free immunosensor array using single-chain antibody fragments. Proc. Natl. Acad. Sci. USA 2005, 10, 14587-14592.

5. Zhang, J.; Lang, H.P.; Huber, F.; Bietsch, A.; Grange, W.; Certa, U.; McKendry, R.; Guntgerodt, H.J.; Hegner, M.; Gerber, C. Rapid and label-free nanomechanical detection of biomarker transcripts in human RNA. Nat. Nanotechnol. 2006, 1, 214-220.

6. Johnson, B.N.; Mutharasan, R. Biosensing using dynamic-mode cantilever sensors: a review. Biosens. Bioelectron. 2012, 32, 1-18.

7. Ilic, B.; Czaplewski, D.; Craighead, H.G.; Neuzil, P.; Campagnolo, C.; Batt, C. Mechanical resonant immunospecific biological detector. Appl. Phys. Lett. 2000, 77, 450-452.

8. Savran, C.A.; Knudsen, S.M.; Ellington, A.D.; Manalis, S.R. Micromechanical detection of proteins using aptamer-based receptor molecules. Anal. Chem. 2004, 76, 3194-3198.

9. Yoo, Y.K.; Chae, M.S.; Kang, J.Y.; Kim, T.S.; Hwang, K.S.; Lee, J.H. Multifunctionalized Cantilever Systems for Electronic Nose Applications. Anal. Chem. 2012, 84, 8240-8245.

10. Alfeeli, B.; Cho, D.; Ashraf-Khorassani, M.; Taylor, L.T.; Agah, M. MEMS-based multi-inlet/outlet preconcentrator coated by inkjet printing of polymer adsorbents. Sens. Actuators B Chem. 2008, 133, 24-32.

11. Camara, E.H.M.; Breuil, P.; Briand, D.; de Rooij, N.F.; Pijolat, C. A micro gas preconcentrator with improved performance for pollution monitoring and explosives detection. Anal. Chim. Acta 2011, $688,175-182$. 
12. Mertens, J.; Finot, E.; Thundat, T.; Fabre, A.; Nadal, M.H.; Eyraud, V.; Bourillot, E. Effects of temperature and pressure on microcantilever resonance response. Ultramicroscopy 2003, 97, 119-126.

13. Sandberg, R.; Svendsen, W.; Molhave, K.; Boisen, A. Temperature and pressure dependence of resonance in multi-layer microcantilevers. J. Micromech. Microeng. 2005, 15, 1454-1458.

14. Finot, E.; Passian, A.; Thundat, T. Measurement of mechanical properties of cantilever shaped materials. Sensors 2008, 8, 3497-3541.

15. Lee, J.H.; Yoon, K.H.; Hwang, K.S.; Park, J.; Ahn, S.; Kim, T.S. Label free novel electrical detection using micromachined PZT monolithic thin film cantilever for the detection of C-reactive protein. Biosens. Bioelectron. 2004, 20, 269-275.

16. Alfeeli, B.; Taylor, L.T.; Agah, M. Evaluation of Tenax TA thin films as adsorbent material for micro preconcentration applications. Microchem. J. 2010, 95, 259-267.

17. Sigman, M.E.; Ma, C.Y.; Ilgner, R.H. Performance evaluation of an in-injection port thermal desorption/gas chromatographic/negative ion chemical ionization mass spectrometric method for trace explosive vapor analysis. Anal. Chem. 2001, 73, 792-798.

18. Kim, S.K.; Chang, H.W.; Zellers, E.T. Microfabricated Gas Chromatograph for the Selective Determination of Trichloroethylene Vapor at Sub-Parts-Per-Billion Concentrations in Complex Mixtures. Anal. Chem. 2011, 83, 7198-7206.

19. De Jager, L.S.; Andrews, A.R.J. Development of a screening method for cocaine and cocaine metabolites in urine using solvent microextraction in conjunction with gas chromatography. J. Chromatogr. A 2001, 911, 97-105.

20. Tian, W.C.; Pang, S.W.; Lu, C.J.; Zellers, E.T. Microfabricated preconcentrator-focuser for a microscale gas chromatograph. J. Microelectromech. Syst. 2003, 12, 264-272.

21. García-Romeo, D.; Pellejero, I.; Urbiztondo, M.A.; Sesé, J.; Pina, M.P.; Martínez, P.A.; Calvo, B.; Medrano, N. Portable low-power electronic interface for explosive detection using microcantilevers. Sens. Actuators B Chem. 2014, 200, 31-38.

22. Mohsen, Y.; Lahlou, H.; Sanchez, J.B.; Berger, F.; Bezverkhyy, I.; Weber, G.; Bellat, J.P. Development of a micro-analytical prototype for selective trace detection of orthonitrotoluene. Microchem. J. 2014, 114, 48-52.

23. Frenois, C.; Barthet, C.; Pereira, F.; Minot, B.; Veignal, F.; Besnard, S.; Rousier, R.; Mayoue, A. Detection of vapour explosives by a multi-sensor prototype-performance evaluation under laboratory and real conditions. In Proceedings of the 2014 IEEE Sensors, Valencia, Spain, 2-5 November 2014; pp. 1057-1060.

(C) 2015 by the authors; licensee MDPI, Basel, Switzerland. This article is an open access article distributed under the terms and conditions of the Creative Commons Attribution license (http://creativecommons.org/licenses/by/4.0/). 